\title{
A survey on the distribution of healthy people with different anti-tumour ability
}

Yanhong Gao', Xiaolan $\mathrm{Xu}^{2}$, Zhennan Dong ${ }^{1}$, Chaoguang Jiang ${ }^{3}$, Jin $\mathrm{GaO}^{2}$, Jinchuan $\mathrm{Hu}^{1}$, Junhao Gui ${ }^{1}$, Haibao Wang ${ }^{4}$, Yaping Tian ${ }^{1}$

\begin{abstract}
1Department of Clinical Biochemistry, Chinese PLA General Hospital, China 2Institute of Biophysics of the Chinese Academy of Sciences, Beijing, China ${ }^{3}$ Department of Medical Administration, Chinese PLA General Hospital, China ${ }^{4}$ Department of Transfusion, Chinese PLA General Hospital, China
\end{abstract}

Grant support: Beijing Capital Development Grant, No. 2007-2043 and Chinese PLA General Hospital Science Research Grant, No. 08CXLXB01.

Submitted: 26 January 2010

Accepted: 8 March 2010

Arch Med Sci 2010; 6, 5: 806-814

DOI: 10.5114/aoms.2010.17099

Copyright (c) 2010 Termedia \& Banach

\section{Abstract}

Introduction: The aim of the study was to explore the distribution of healthy people with different anti-tumour ability.

Material and methods: Leukocytes were separated by the Ficoll-Hypaque density gradient centrifugal method. Then they were mixed with A549, MCF-7 and Hela cells at different ratios. The survival rate for target cells was observed and counted by Fluoroskan. Immune function for 200 healthy people was analysed by flow cytometry.

Results: The results obtained by confocal microscopy revealed that human blood leukocytes possessed direct anti-tumour activity. The survival rate for tumour cells was the lowest in the condition of 20:1 ratio of effector cells to target cells. We speculated that in 200 healthy people the leukocyte capacity for killing MCF-7 cells is stronger than the leukocyte capacity for killing A549 cells and Hela cells. We also found that the distribution for 200 healthy people with different anti-tumour ability was different for different tumour cells. The number of healthy people with the strongest anti-tumour ability was highest when the target cells were MCF-7 cells. Moreover, the survival of A549, MCF-7 and Hela cells was correlated with T, B and NK lymphocytes.

Conclusions: From the above, we can select healthy individuals with strong antitumour ability as anti-tumour donors according to their distribution with different anti-tumour ability, which opened up a new direction for fighting human cancer.

Key words: innate immunity, tumour, leukocytes, polymorphonuclear leucocytes, distribution, healthy people.

\section{Introduction}

Cancer is one of the most serious diseases that threaten human beings today. More than 5 million people each year die of multiple types of cancer, including liver, lung, breast, prostate cancers, etc. At present, the incidence of all kinds of cancer (both squamous cell and adenocarcinoma) appears to be increasing. It is estimated that the number of cancer deaths globally each year could double by 2020.

There are many tumours resisting conventional chemotherapy and radiotherapy, and at present these tumours indicate a poor prognosis.

\author{
Corresponding author: \\ Yaping Tian \\ Department of Clinical \\ Biochemistry \\ Chinese PLA General Hospital \\ 28 Fuxing Road \\ Beijing 100853, China \\ Phone: +8610 66939374 \\ Fax: +8610 88217385 \\ E-mail: tianyp61@gmail.com
}


Thus more effective immunotherapy is needed, especially for advanced tumours. There are two functional systems in tumour immunotherapy: innate immunity and acquired immunity [1-4]. Innate immunity, also known as natural immunity, is the condition where the body has the inherent ability for resistance against invasion of pathogens and removal of foreign bodies in vivo. Innate immunity involves a large number of different cell populations such as epithelial cells, monocytes, macrophages, dendritic cells, polymorphonuclear leucocytes (PMN), and natural killer (NK) cells. The traditional view supported the idea that innate immunity and acquired immunity (i.e., adaptive immunity, specific immunity) represented two separate types of immunity. They have different evolution, development, mechanism of action and characteristics. Innate immunity is the first protective line in resisting the pathogenic microorganism. It can initiate and participate in the acquired immune response, and acquired immunity may produce identification and subsequent activity against specific pathogens (antigens) in long-term exposure to exogenous pathogens. Due to its specific characteristics, such as memory, the acquired immune response has always been regarded as the body's main anti-tumour immune response mechanism. However, innate immunity has been studied widely in an anti-infective field instead of an anti-cancer field due to its instantaneous character and lack of memory, which is different from acquired immunity.

Recently, Cui et al. discovered that SR/CR mice have these abilities with resistance to S180-induced ascites (spontaneous regression, SR) or remained ascites-free after repeated injections of S180 cells (complete resistance, CR) [5]. Further study has shown that macrophages, polymorphonuclear leucocytes (PMN) and natural killer cells play an important role in attacking tumour cells [5-8].

The PMN, monocyte-macrophages and NK cells belong to innate immune cells. Although they start to participate in and regulate the acquired immune response by assisting $T$ lymphocytes to recognize tumour cells, they themselves have a certain antitumour capacity. It has been reported that this capability is inherent and does not vary from person to person. In a series of anti-tumour studies on SR/CR mice, Cui found that this natural anti-tumour ability is not only genetic, but is also transferred, which provides a new strategy for tumour immunotherapy.

Can we discover the same cancer-resistance trait in leukocytes of healthy people? How do we obtain their immune cells? How do we choose the best cancer-resistant people? Will individual differences for healthy people result in anti-tumour ability differences among them? Is the difference in cancer resistance related to the $T$ lymphocyte acquired immunity?

In order to answer the above questions, we analysed blood samples from 200 healthy people and explored the distribution of healthy people with different anti-tumour ability in order to open up a new direction for fighting human cancer.

\section{Material and methods}

\section{Cell lines}

A549, MCF-7 and Hela cell lines were purchased from the American Type Culture Collection and propagated in DMEM (Dulbecco's Modified Eagle's Medium, Gibco) supplemented with $10 \%$ heatinactivated fetal bovine serum (FBS), 100 units/ml penicillin and $100 \mathrm{mg} / \mathrm{ml}$ streptomycin. Cultures were maintained at $37^{\circ} \mathrm{C}$ in a humidified atmosphere containing $95 \%$ air and 5\% carbon dioxide.

\section{Isolation of human peripheral blood leukocytes and their percentage analyses}

Blood samples of 200 healthy people were provided by the Chinese PLA General Hospital. The fresh blood samples were first diluted 1:1 with phosphate-buffered saline (PBS) and then added to a Ficoll-Hypaque density gradient solution. The suspension was centrifuged at $400 \mathrm{~g}$ for $15 \mathrm{~min}$ at room temperature. PMN were harvested and mixed with $2 \mathrm{ml}$ of $\mathrm{H}_{2} \mathrm{O}$ and $1 \mathrm{ml}$ of PBS for $10 \mathrm{~min}$ to destroy red blood cells. The suspension was then centrifuged at $400 \mathrm{~g}$ for $3 \mathrm{~min}$ at RT. Cells were resuspended in PBS and the cell concentration was adjusted at a density of $2.5 \times 10^{5} / \mathrm{ml}$. Then, they were labelled with specific anti-CD45 (Beckman) to the cell surface according to standard procedures recommended by the manufacturer and analysed on a Beckman Coulter EPICS XL (Beckman Coulter, Miami FL, USA).

\section{Tumour cells labelled with CFSE}

A $5 \mathrm{mM}$ stock solution of carboxyfluorescein succinimidyl ester (CFSE) (Invitrogen) was prepared by dissolving $500 \mu \mathrm{g}$ of CFSE into $180 \mu$ l of dimethyl sulfoxide. Tumour cells were plated at a density of $8 \times 10^{4}$ cells per well in 24 -well plates. When they were grown overnight to $80 \%$ confluence, tumour cells were suspended in PBS/0.1\% BSA at a density of $1 \times 10^{6} \mathrm{cells} / \mathrm{ml}$. Then they were incubated with $2 \mu \mathrm{l}$ of $5 \mathrm{mM} \mathrm{CFSE}$ at $37^{\circ} \mathrm{C}$ for $10 \mathrm{~min}$, which resulted in a final concentration of $10 \mu \mathrm{M}$. The reaction was stopped by adding $5 \mathrm{~V}$ ice-cold culture medium. They were centrifuged and washed twice with ice-cold PBS. After that, they were observed under a laser scanning confocal microscope. 


\section{In vitro assays of target cell survival rate}

Tumour cells labelled with CFSE were seeded in 96-well plates, and then leukocytes were added. The effector cells were mixed with target cells at a ratio of $5: 1,10: 1$ and $20: 1$, and they were incubated for $48 \mathrm{~h}$ at $37^{\circ} \mathrm{C}$ in a humidified atmosphere containing 95\% air and 5\% carbon dioxide. The survival rate for target cells was observed and counted by Fluoroskan.

\section{Immune function analyses by flow cytometry}

Whole blood samples were taken using sterile tubes with EDTA anticoagulant. First, $10 \mu \mathrm{l}$ of CD45FITC/CD4-RD1/CD8-ECD/CD3-PC5 or CD45-FITC/ CD56-RD1/C19-ECD/CD3-PC5 was added to each sample label test tube, and $100 \mu \mathrm{l}$ of whole blood samples were added to it after adjusting the white cell count to approximately $5 \times 10^{9}$ cells/l. Then they were incubated in the dark at room temperature for $20 \mathrm{~min} 500 \mu \mathrm{l}$ of Optilyse C lysing solution was added to whole blood samples and vortex mixed. They were incubated at room temperature for

\section{A}

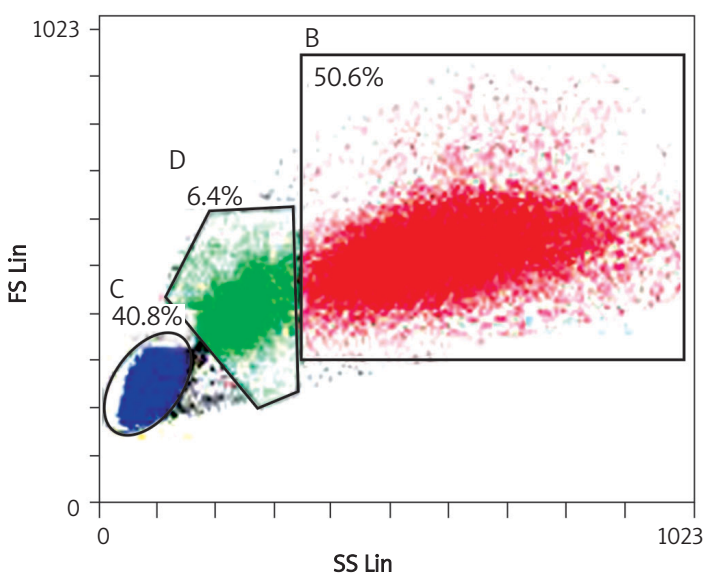

B

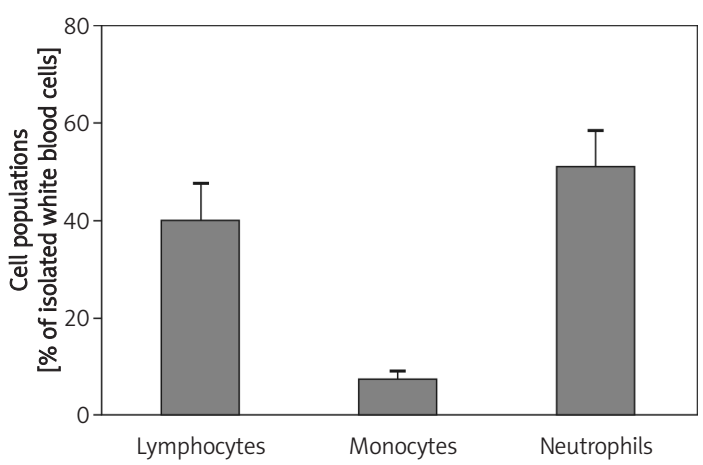

Figure 1. Flow cytometric analysis of peripheral blood from healthy people after CD45 labelling. The results demonstrated that lymphocytes, monocytes and neutrophils can be divided well by CD45 labelling (A). The percentage of lymphocytes is $40.02 \%$, monocytes $7.30 \%$, neutrophils $51.18 \%$ (B)
10 min. Finally, $500 \mu$ l of PBS was added followed by brief vortex mixing. After $10 \mathrm{~min}$, cells were analysed by flow cytometry.

\section{Statistical analysis}

All experiments were repeated a minimum of three times. The data are presented as means \pm SD. Statistics were calculated using SPSS 10.0 software for all analyses. Statistical differences between the two groups were analysed using the $t$ test. $P$ values less than 0.05 were considered significant.

\section{Results}

The analysis of leukocyte subsets and purity by flow cytometry

To determine the cell separated effect using the Ficoll-Hypaque density gradient centrifugal method, leukocyte subsets and purity from peripheral blood of healthy people were analysed by flow cytometry. The results demonstrated that three groups of cells can be divided well by CD45 labelling. They were lymphocytes, monocytes, and neutrophils (Figure 1. A). The percentage of lymphocytes was $40.02 \%$, monocytes $7.30 \%$ and neutrophils $51.18 \%$ (Figure 1. B).

\section{In vitro assays of target cells' survival rate}

To address the question of whether human leukocytes have the cancer-resistance trait, like the SR/CR mouse model, we isolated leukocytes from healthy people and mixed them with A549 (human lung carcinoma epithelial cell line), MCF-7 (human breast adenocarcinoma cell line) and Hela (cervix adenocarcinoma epithelial cell line) cells at different ratios. The survival rate for target cells was observed and counted by Fluoroskan.

The results showed that the survival rate for tumour cells was different for different ratios of effector cells to target cells (Figures 2-4). For A549 cells, we found that the survival rate for A549 cells decreased when the ratio of effector cells to target cells increased. When the ratio of effector cells to target cells was $5: 1$, it was not obviously different than control. In the condition of a $20: 1$ ratio of effector cells to target cells, the survival rate for A549 cells was the lowest. From Figure 2. D, we can see A549 cells' lysis by infiltrating leukocytes. The same results were obtained from Hela and MCF-7 cells.

\section{Target cells' survival rate statistics at different ratios of effector cells to target cells}

The effector cells are leukocytes from peripheral blood of healthy people which were isolated by Ficoll-Hypaque density gradient solution. The target cells were A549, MCF-7 and Hela cells. The target 

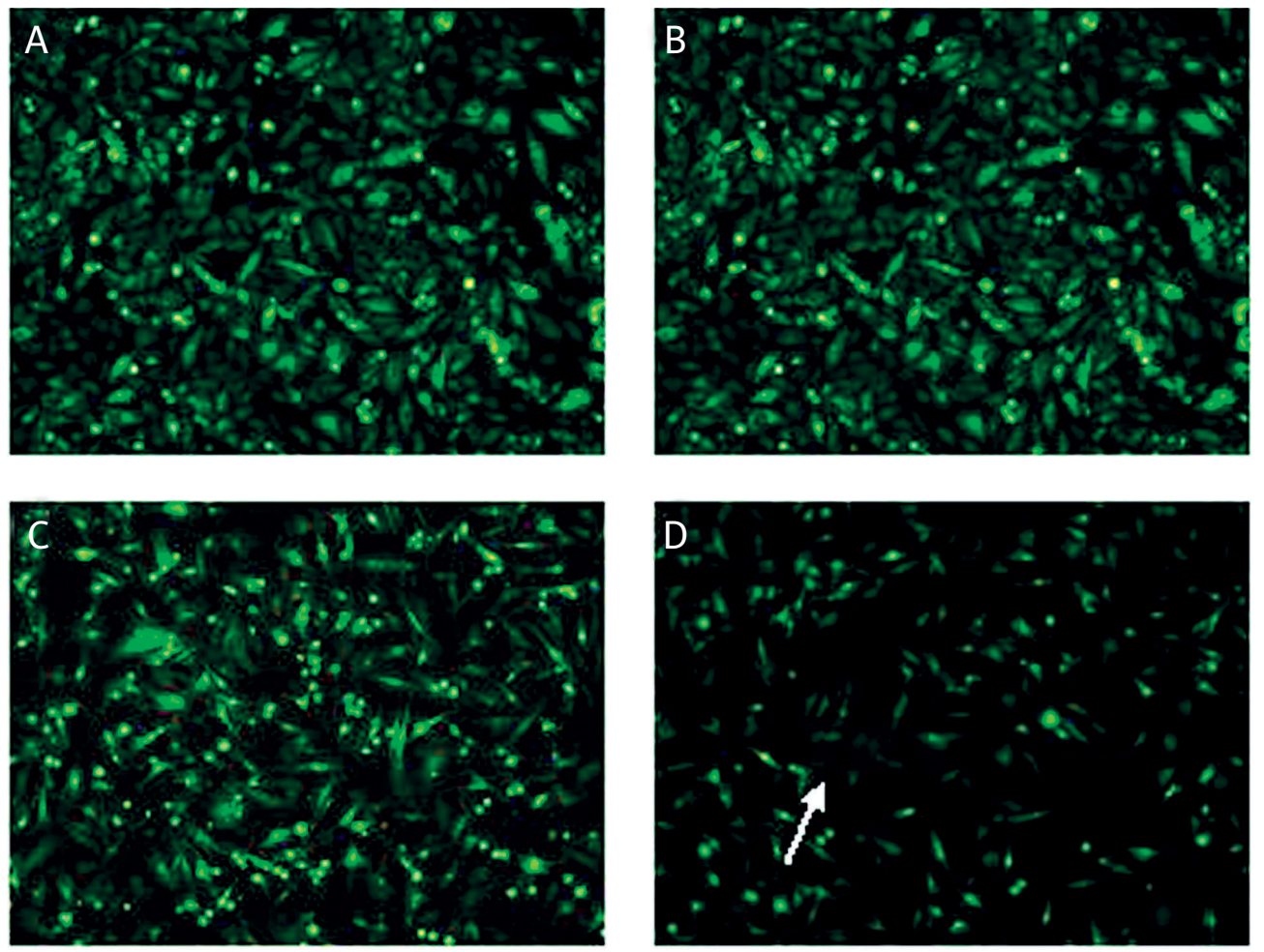

Figure 2. Survival rate for A549 cells. After separating 200 blood donors' leukocytes with the Ficoll-Hypaque density gradient method, tumour cells labelled with CFSE were mixed with leukocytes at different ratios and incubated for $48 \mathrm{~h}$ at $37^{\circ} \mathrm{C}$. The images were obtained with confocal microscopy (Fluoroskan). (A) A549 cells labelled with CFSE; (B) The ratio of effector cells to A549 cells was $5: 1$; (C) The ratio of effector cells to A549 cells was $10: 1$; (D) The ratio of effector cells to A549 cells was $20: 1$. Scale bars: $50 \mu \mathrm{m}$
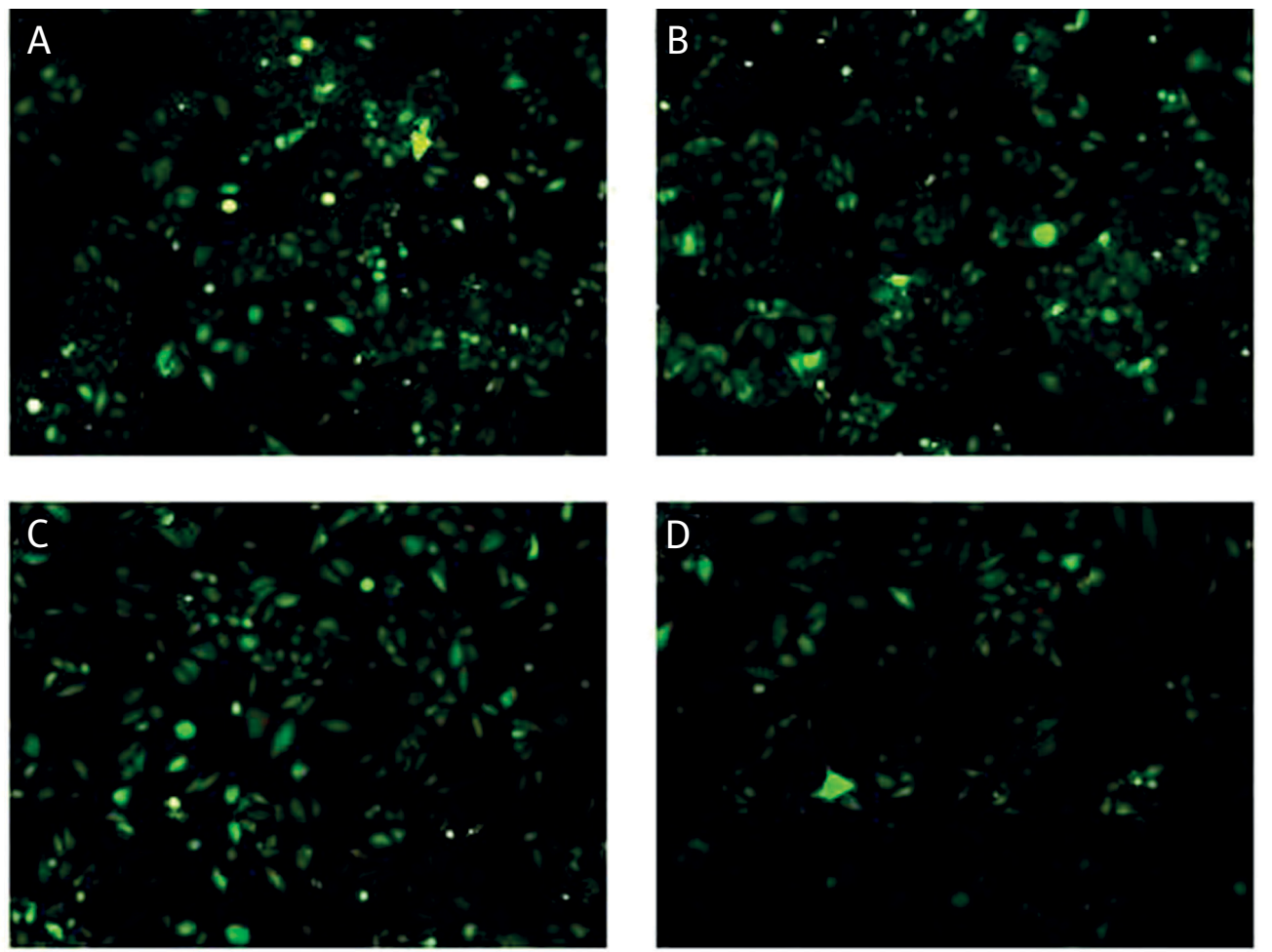

Figure 3. Survival rate for Hela cells. After separating 200 blood donors' leukocytes with the Ficoll-Hypaque density gradient method, tumour cells labelled with CFSE were mixed with leukocytes at different ratios and incubated for $48 \mathrm{~h}$ at $37^{\circ} \mathrm{C}$. The images were obtained with confocal microscopy (Fluoroskan). (A) Hela cells labelled with CFSE;

(B) The ratio of effector cells to Hela cells was $5: 1$; (C) The ratio of effector cells to Hela cells was $10: 1$; (D) The ratio of effector cells to Hela cells was $20: 1$. Scale bars: $50 \mu \mathrm{m}$ 

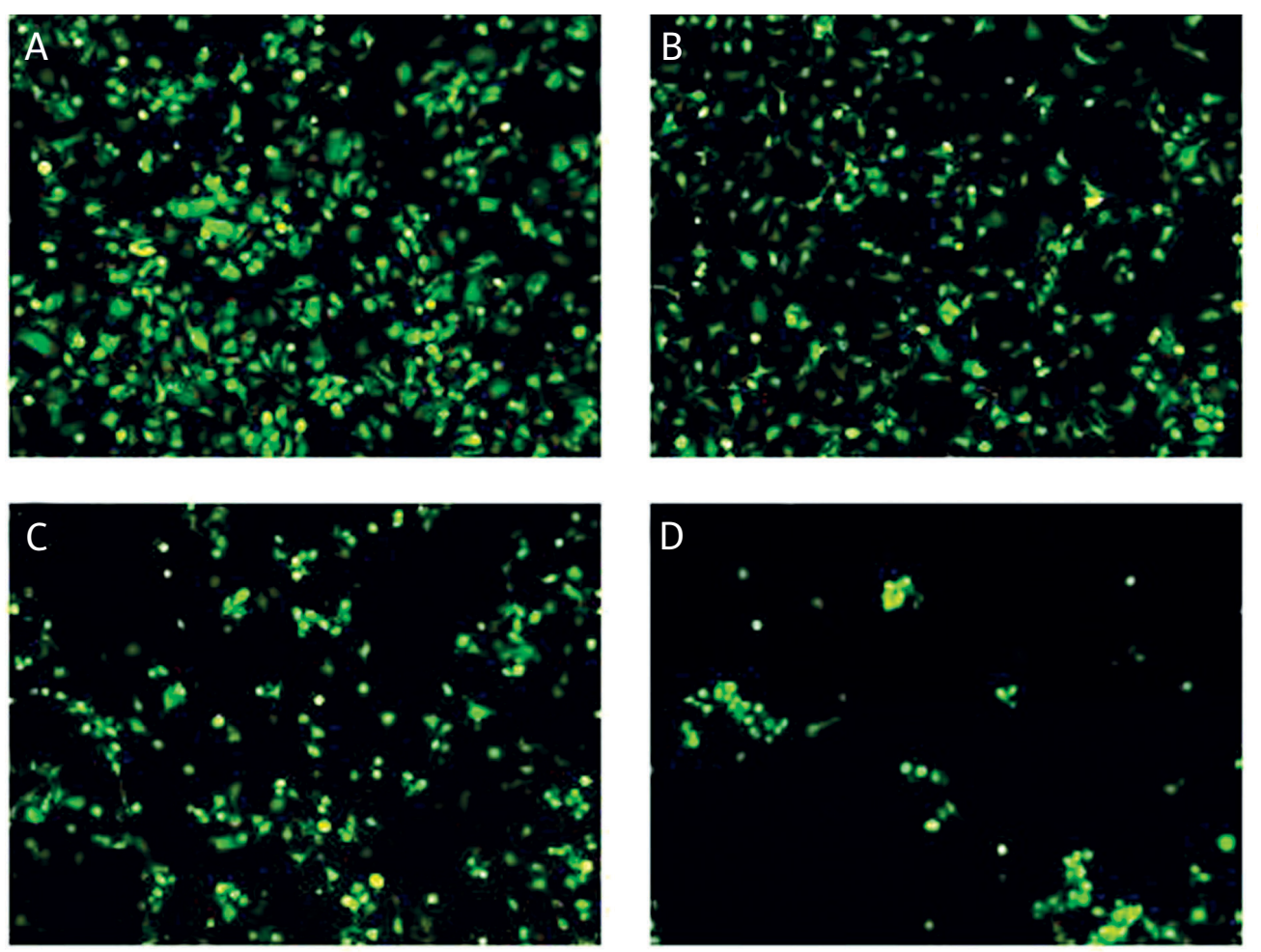

Figure 4. Survival rate for MCF-7 cells. After separating 200 blood donors' leukocytes with the Ficoll-Hypaque density gradient method, tumour cells labelled with CFSE were mixed with leukocytes at different ratios and incubated for $48 \mathrm{~h}$ at $37^{\circ} \mathrm{C}$. The images were obtained with confocal microscopy (Fluoroskan). (A) MCF-7 cells labelled with CFSE; (B) The ratio of effector cells to MCF-7 cells was $5: 1$; (C) The ratio of effector cells to MCF-7 cells was 10:1; (D) The ratio of effector cells to MCF-7 cells was $20: 1$. Scale bars: $50 \mu \mathrm{m}$

cells were labelled with CFSE as above. Then the effector cells were mixed with target cells at different ratios. They were incubated for $48 \mathrm{~h}$ at
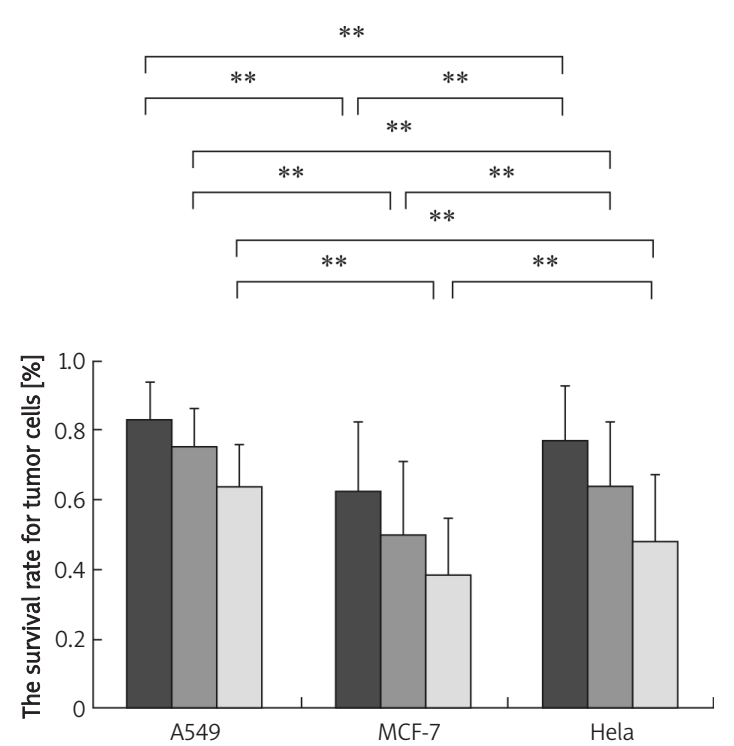

Tumor cells at different ratio with effector cells to target cells
$\square$ 5:01
$\square 10: 01$

$\square 20: 01$ $37^{\circ} \mathrm{C}$. To evaluate the effect of leukocytes killing target cells, we counted the survival rate of target cells at different ratios of effector cells to target cells.

The results showed that the survival rate for target cells was the lowest in the condition of a $20: 1$ ratio of effector cells to target cells. The survival rate for $\mathrm{A} 549$ was $0.64 \pm 0.12$. The survival rate for MCF-7 cell was $0.38 \pm 0.17$ (Figure 5). From the above, we can speculate that in 200 healthy people the capacity of leukocytes for killing MCF-7 cells is stronger than the capacity of leukocytes for

Figure 5. The survival rate for target cells was counted by Fluoroskan at different ratios for effector cells and target cells. The results indicated that the survival rate for tumour cells was different for different effector cells with the same ratio $\left({ }^{\star *} P<0.01\right)$. In the condition of a $20: 1$ ratio of effector cells to target cells, the survival rate for tumour cells was the lowest. The survival rate for A549 was $0.64 \pm 0.12$. The survival rate for MCF-7 cells was $0.38 \pm 0.17$. This showed that the MCF-7 cell killing capacity of leukocytes is stronger than the killing capacity for A549 cells. Leukocytes from healthy people act differently in their effect on different tumour cells 
killing A549 cells. This is consistent with the results of Fig. 2-4. Furthermore, leukocytes of healthy people act differently in their effect on different tumour cells.

\section{Distribution of 200 healthy people with different anti-tumour ability}

For A549, MCF-7 and Hela cells, in the condition of a $20: 1$ ratio of effector cells to target cells, the distribution of 200 healthy people with different anti-tumour ability is shown in Figure 6. In accordance with their different capacity for killing tumour cells, the 200 healthy people were divided into three groups, of which group $A$ has the strongest anti-tumour ability (tumour cell survival rate $0-30 \%$ ), group B has medium anti-tumour ability (tumour cell survival rate 30-70\%), and group $\mathrm{C}$ has the weakest anti-tumour ability (tumour cell survival rate $70-100 \%)$.

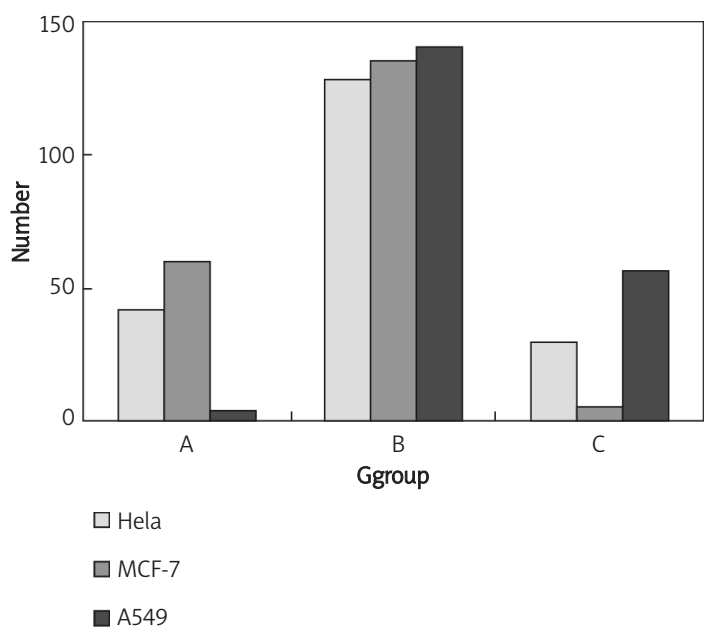

Figure 6. Distribution of 200 healthy people with different capacity for killing tumour cells. Group A has the strongest anti-tumour ability (tumour cells' survival rate is in the range $0-30 \%$ ), group $B$ has medium anti-tumour ability (tumour cells' survival rate $30-70 \%$ ), and group C has the weakest antitumour ability (tumour cells' survival rate $70-100 \%$ )
For A549 cells, among 200 healthy people, the number of healthy people with the strongest antitumour ability was 4; the number of healthy people with medium anti-tumour ability was 140; the number of healthy people with the weakest antitumour ability was 56.

For MCF-7 cells, the number of healthy people with the strongest anti-tumour ability was 60; the number of healthy people with medium anti-tumour ability was 135; the number of healthy people with the weakest anti-tumour ability was 5 .

For Hela cells, the number of healthy people with the strongest anti-tumour ability was 42; the number of healthy people with medium anti-tumour ability was 128; the number of healthy people with the weakest anti-tumour ability was 30 .

\section{Percentage analysis of 200 healthy people with different anti-tumour ability}

For A549 cells, among 200 healthy people, the percentage of healthy people with the strongest anti-tumour ability was $2 \%$; the percentage of healthy people with medium anti-tumour ability was $70 \%$; the percentage of healthy people with the weakest anti-tumour ability was $28 \%$.

For MCF-7 cells, the percentage of healthy people with the strongest anti-tumour ability was 30\%; the percentage of healthy people with medium antitumour ability was $67 \%$; the percentage of healthy people with the weakest anti-tumour ability was $3 \%$.

For Hela cells, the percentage of healthy people with the strongest anti-tumour ability was $21 \%$; the percentage of healthy people with medium antitumour ability was $64 \%$; the percentage of healthy people with the weakest anti-tumour ability was 15\% (Figure 7).

Comparison analysis of target cell survival rate following leukocyte infiltration for 200 healthy people of different ages

The 200 healthy people were divided into group $A$ and group $B$ according to their ages. The age for
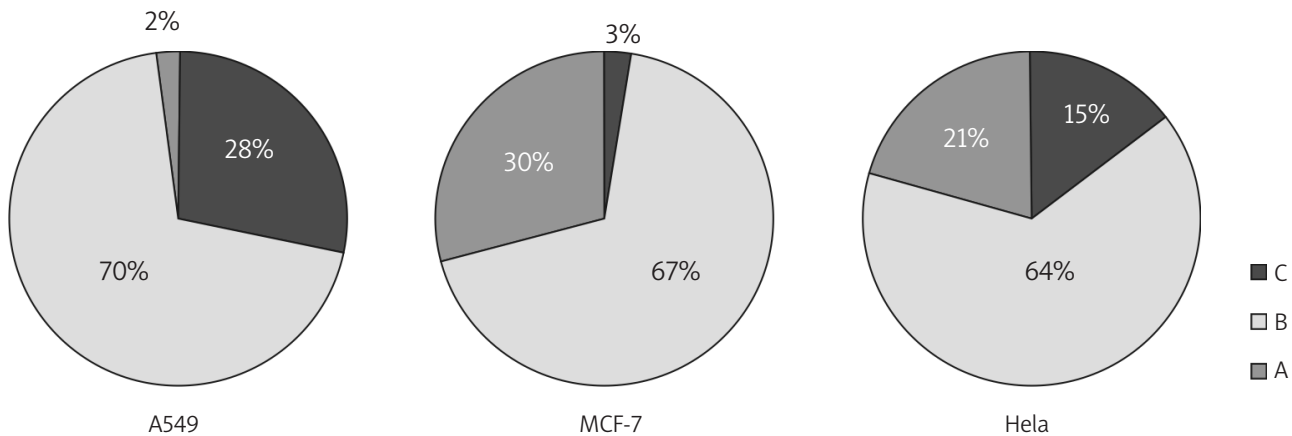

Figure 7. Percentage analysis of 200 healthy people with different capacity for killing tumour cells. Group A has the strongest anti-tumour ability (tumour cells' survival rate $0-30 \%$ ), group B has medium anti-tumour ability (tumour cells' survival rate 30-70\%), and group C has the weakest anti-tumour ability (tumour cells' survival rate $70-100 \%$ ) 
Table I. Comparison analysis of target cells' survival rate after leukocyte infiltration in 200 healthy people of different age groups

\begin{tabular}{|ccccc|}
\hline & $N$ & A549 & MCF-7 & Hela \\
\hline Group A & 35 & $0.61 \pm 0.11$ & $0.37 \pm 0.13$ & $0.53 \pm 0.15^{*}$ \\
\hline Group B & 106 & $0.64 \pm 0.13$ & $0.38 \pm 0.18$ & $0.46 \pm 0.18$ \\
\hline Group C & 59 & $0.65 \pm 0.11$ & $0.40 \pm 0.16$ & $0.49 \pm 0.21$ \\
\hline
\end{tabular}

Comparing with group $B,{ }^{\star} P<0.05$

group A was below 20 years, the age for group B was in the range of 20-25 years, and the age for group C was over 25 years. As shown in Table I, no difference was observed in the survival rate for A549 cells and MCF-7 cells among 200 healthy people in different age groups. For Hela cells, the difference in cell survival rate was significant between group $\mathrm{A}$ and group $\mathrm{B}(P<0.05)$.

Correlation analysis between target cell survival rate following leukocyte infiltration in 200 healthy people and immune function of healthy people

In order to investigate whether the tumour cell killing ability of healthy people was related to their immune function, we analysed the correlation between target cells' survival rate after leukocyte infiltration in 200 healthy people and immune function in healthy people.

As shown in Table II, the value of $\mathrm{CD}^{+}{ }^{+} \mathrm{CD} 8^{+} \mathrm{T}$ lymphocytes was positively related to survival rate of A549 cells $(r=0.172, P<0.05)$; the value of $\mathrm{CD}^{+}{ }^{+} \mathrm{CD} 4^{+} \mathrm{T}$ lymphocytes was negatively correlated with survival rate of $\mathrm{A} 549$ cells $(r=-0.189, P<0.01)$; the value of CD4/CD8 was negatively related to survival rate of $A 549$ cells $(r=-0.188, P<0.01)$; the values of $\mathrm{CD}^{+}, \mathrm{CD}^{+}{ }^{+} \mathrm{CD} 4^{+}, \mathrm{CD} 4 / \mathrm{CD} 8, \mathrm{CD} 3-\mathrm{CD} 56^{+} \mathrm{T}$ lymphocytes were positively related to survival rate of MCF-7 cells; $\mathrm{CD}^{-}{ }^{-} \mathrm{CD} 56^{+} \mathrm{B}$ lymphocytes were negatively related to survival rate of MCF-7 cells. The value of $\mathrm{CD}^{-} \mathrm{CD}^{-} 9^{+}, \mathrm{CD}^{-} \mathrm{CD} 56^{+} \mathrm{B}$ lymphocytes was negatively related to survival rate of Hela cells.

These findings showed that survival rate of A549, MCF-7 and Hela cells was correlated with T, B and NK lymphocytes.

\section{Discussion}

The concept of using immune cells from one person to fight cancer in another person is a very pertinent topic right now. Dr Cui's findings suggested that it may be possible to transfer the ability to fight cancer between mice. Cui's group also successfully treated a range of different cancers in mice by injecting them with granulocytes from a strain of mice that are completely resistant to cancer.

All of us have some ability to fight cancer, via immune cells which can identify and kill tumour cells, although the extent of these cells' influence is not known. Can we discover the same cancerresistance trait in leukocytes of healthy people? How do we get their immune cells? How do we choose the best cancer-resistant people in healthy people? In order to answer this question, we investigated the distribution of 200 healthy people with different anti-tumour ability.

We separated leukocyte cells using the FicollHypaque density gradient centrifugal method first, and analysed them by flow cytometry. The percentage of lymphocytes was $40.02 \%$, monocytes $7.30 \%$, and PMN $51.18 \%$. Then, we mixed the separated cells with tumour cells labelled with CFSE at different ratios and incubated them for $48 \mathrm{~h}$ at $37^{\circ} \mathrm{C}$. The survival rate for tumour cells was observed and counted by Fluoroskan.

The confocal microscopy results revealed that human blood leukocytes possessed direct antitumour activity. Further studies suggested that the survival rate for tumour cells was the lowest in the

Table II. Correlation analysis between target cell survival rate following leukocyte infiltration in 200 healthy people and immune function $(n=200)$

\begin{tabular}{|c|c|c|c|c|c|c|c|}
\hline & $\mathrm{E} / \mathrm{T}$ & $\mathrm{CD}^{+}$ & $\mathrm{CD}^{+} \mathrm{CD}^{+}$ & $\mathrm{CD}^{+} \mathrm{CD}^{+}$ & CD4/CD8 & $\mathrm{CD}^{-} \mathrm{CD}^{-} 9^{+}$ & $\mathrm{CD}^{-} \mathrm{CD}^{-} 6^{+}$ \\
\hline \multirow[t]{3}{*}{ A549 } & $5: 1$ & 0.107 & -0.019 & $0.172^{*}$ & -0.072 & -0.065 & -0.096 \\
\hline & $10: 1$ & 0.068 & 0.015 & 0.084 & -0.107 & -0.007 & -0.111 \\
\hline & $20: 1$ & -0.075 & $-0.189^{\star *}$ & 0.073 & $-0.188^{\star \star}$ & -0.087 & 0.047 \\
\hline \multirow[t]{3}{*}{ MCF-7 } & $5: 1$ & $0.178^{\star}$ & $0.195^{\star *}$ & 0.022 & $0.201^{\star *}$ & -0.079 & $-0.211^{\star *}$ \\
\hline & $10: 1$ & $0.155^{\star}$ & $0.175^{*}$ & -0.011 & $0.144^{*}$ & -0.053 & $-0.151^{\star}$ \\
\hline & $20: 1$ & 0.097 & 0.113 & -0.030 & 0.054 & -0.088 & -0.079 \\
\hline \multirow[t]{3}{*}{ Hela } & $5: 1$ & 0.105 & 0.110 & 0.013 & 0.110 & -0.032 & $-0.159^{\star}$ \\
\hline & $10: 1$ & 0.115 & 0.093 & 0.021 & 0.113 & -0.125 & $-0.208^{\star}$ \\
\hline & $20: 1$ & 0.123 & 0.074 & 0.068 & 0.070 & $-0.140^{\star}$ & $-0.210^{\star}$ \\
\hline
\end{tabular}

${ }^{\star} P<0.05,{ }^{* *} P<0.01$ 
condition of a $20: 1$ ratio of effector cells to target cells.

We found that the distribution for 200 healthy people with different anti-tumour ability was different for different tumour cells. For A549 cells, among 200 healthy people, the percentage of healthy people with the strongest anti-tumour ability was $2 \%$; the percentage of healthy people with the strongest anti-tumour ability was 30\% and $21 \%$ for MCF-7 cells and Hela cells, respectively. This showed that the number of healthy people with the strongest anti-tumour ability was highest when the target cells were MCF-7 cells.

The innate immune response is known to play a critical role in the development of effective antitumour immunity and granulocytes, such as polymorphonuclear neutrophils (PMNs). As key mediators of inflammation, they have been suggested to have the potential to initiate immune response cascades against tumours. So, we speculated that the distribution for 200 healthy people with different antitumour ability was related to the anti-tumour effects of PMN. Many studies have confirmed that the antitumour effects of hyperthermia and BCG are related to the site of PMN infiltration [9-10]. PMN around the tumour can kill the tumour by releasing a series of cytotoxic substances, such as reactive oxygen species, nitric oxide, lysosome, IL-1, TNF and other cytokines. PMN was found in vitro to have a certain role in antitumour activity [11-13].

Another study showed PMN having close contacts with injured tumour cells, and activated PMN may adhere to the surface of tumour cells and cleave them. At the same time, degranulation of PMN occurred. In Cui's research on SR/CR mice, they did not produce ascites by intraperitoneal injection of S180 tumour cells. The reason is that PMN of mice can rapidly reach the periphery of tumour cells and S180 was surrounded by PMN, macrophage and NK cells, which formed a rosette [11-12]. By electron microscopy observation, they found that the microvilli in the surface of tumour cells reduce and damage cell membranes, which induce death of tumour cells. We support the direct anti-tumour activity mechanism which was observed in human leukocytes in relation to the above discussion.

PMN fight tumours not only through the direct killing of tumour cells, but also through antibodydependent cell-mediated cytotoxicity (ADCC) [14-15]. Research has shown that early rupture of tumour cells has been correlated with a PMN-mediated ADCC role. The application of granulocyte-macrophage colony-stimulating factor (GM-CSF) can increase the role of PMN in ADCC. Pre-clinical studies on renal cell carcinoma found that a united application of anti-epidermal growth factor receptor, anti-human leukocyte Fc receptor and myeloid growth factors (such as GM-CSF) may kill a large number of tumour cells, and PMN is the effector cell mediating anti-tumour activity.

Moreover, we found that in different age groups of healthy people the difference in cell survival rate was significant between group $A$ and group $B$ for Hela cells $(P<0.05)$. The survival rate for Hela cells decreased as age increased. This means that the killing rate for Hela cells increased with an increase in age of healthy people $(P<0.01)$. This is not consistent with Dr Cui's reports, because the age of healthy people we chose was between 18 and 23 years and the average age is $25 \pm 7.3$ years. Therefore, further study concerning age needs to be conducted.

Leukocytes of healthy people act differently in their effect on different tumour cells. Is this related to their immune function? We analysed the correlation between target cells' survival rate after the infiltration of leukocytes in 200 healthy people and immune function of healthy people. It showed that survival of A549, MCF-7 and Hela cells was correlated with T, B and NK lymphocytes. This was not the same as the result of Dr Cui, which demonstrated that NK played an important role in the destruction of cancer cells [6].

In summary, our study suggested that human blood leukocytes possess direct anti-tumour activity. The distribution for 200 healthy people with different anti-tumour ability that was different for different tumour cells has been found to possibly be related to the anti-tumour effects of PMN. According to this, we can choose healthy individuals with strong anti-tumour ability as anti-tumour donors according to their distribution with different anti-tumour ability. Future considerations include how to validate strong anti-tumour donors from a crowd of people and how to further activate innate immune cells and how to avoid allogeneic transfusion that may lead to rejection. These need to be addressed in future research work.

\section{Acknowledgments}

Our manuscript concerned clinical studies using human blood samples. The study protocol was approved by the Research Ethics Committee of the Chinese PLA General Hospital and received each healthy person's consent. The authors declare no conflict of interest.

\section{References}

1. Adam JK, Odhav B, Bhoola KD. Immune responses in cancer. Pharmacol Ther 2003; 99: 113-32.

2. Ikeda H, Chamoto K, Tsuji T, et al. The critical role of type1 innate and acquired immunity in tumor immunotherapy. Cancer Sci. 2004; 95: 697-703.

3. Rolle CE, Carrio R, Malek TR. Modeling the CD8+ T effector to memory transition in adoptive T-cell antitumor immunotherapy. Cancer Res 2008; 68: 2984-92. 
4. Wurzenberger C, Koelzer VH, Schreiber S, et al. Short-term activation induces multifunctional dendritic cells that generate potent antitumor T-cell responses in vivo. Cancer Immunol Immunother. 2009; 58: 901-13.

5. Cui Z, Willingham MC, Hicks AM, et al. Spontaneous regression of advanced cancer: identification of a unique genetically determined, age-dependent trait in mice. Proc Natl Acad Sci U S A 2003; 100: 6682-7.

6. Hicks AM, Riedlinger G, Willingham MC, et al. Transferable anticancer innate immunity in spontaneous regression/complete resistance mice. Proc Natl Acad Sci U S A 2006; 103: 7753-8.

7. Cui Z, Willingham MC. The effect of aging on cellular immunity against cancer in SR/CR mice. Cancer Immunol Immunother 2004; 53: 473-8.

8. Hicks AM, Willingham MC, Du W, et al. Effector mechanisms of the anti-cancer immune responses of macrophages in SR/CR mice. Cancer Immun 2006; 6: $11-24$.

9. Ostberg JR, Ertel BR, Lanphere JA, et al. An important role for granulocytes in the thermal regulation of colon tumor growth. Immunol Invest 2005; 34: 259-72.

10. Suttmann H, Riemensberger J, Bentien G, et al. Neutrophil granulo2 cytes are required for effective bacillus calmette - guerin immunotherapy of bladder cancer and orchestrate local immune responses. Cancer Res 2006; 66: 8250-7.

11. Siracusano S, Vita F, Abbate R, et al. The role of granulocytes following intravesical BCG prophylaxis. Eur Urol 2007; 51: 1589-97.

12. Bergami-Santos PC, Mariano M, Barbuto JA. Dual role of polymorphonuclear neutrophils on the growth of Ehrlich ascites tumor (EAT) in mice. Life Sci 2004; 75: 245-55.

13. Alvarez MJ, Prada F, Salvatierra E, et al. Secreted protein acidic and rich in cysteine produced by human melanoma cells modulates polymorphonuclear leukocyte recruitment and antitumor cytotoxic capacity. Cancer Res 2005; 65: 5123-32.

14. Stockmeyer B, Beyer T, Neuhuber W, et al. Polymorphonuclear granulocytes induce antibody - dependent apoptosis in human breast cancer cells. J Immunol 2003, 171: 5124-9.

15. Lum HD, Buhtoiarov IN, Schmidt BE, et al. Tumoristatic effects of anti-CD40 mAb-activated macrophages involve nitric oxide and tumour necrosis factor-alpha. Immunology 2006; 118: 261-70. 